'Departamento de Psicología, Facultad de Educación, Ciencias Sociales y Humanidades, Universidad de La Frontera. Temuco, Chile.

${ }^{2}$ Laboratorio de Estrés y Salud. Facultad de Educación, Ciencias Sociales y Humanidades, Universidad de La Frontera. Temuco, Chile.

${ }^{3}$ Departamento de Medicina Interna, Facultad de Medicina,

Universidad de La Frontera. Temuco, Chile.

${ }^{4}$ Centro de Investigación en Epidemiología Cardiovascular y Nutricional (EPICYN), Universidad de La Frontera. Temuco, Chile. apsicólogo. bPhD.

Esta investigación fue financiada por CONICYT (Comisión Nacional de Investigación Científica y Tecnológica, Gobierno de Chile), Proyecto FONDECYT de INICIACION N¹1140454 cuyo investigador principal fue el Dr. Manuel S. Ortiz.

Recibido el 27 de febrero de 2018, aceptado el 23 de noviembre de 2018

Correspondencia a: Manuel S. Ortiz Departamento de Psicología, Universidad de La Frontera. Montevideo 0830, Temuco, Chile. manuel.ortiz@ufrontera.cl

\section{Estrés psicológico y síndrome metabólico}

\author{
MANUEL S. ORTIZ ${ }^{1,2,4, a, b}$, JORGE SAPUNAR ${ }^{3,4}$
}

\section{Longitudinal association between chronic psychological stress and metabolic syndrome}

Background: Psychological stress and depressive symptoms are variables associated with metabolic syndrome (MetS). Aim: To determine the longitudinal association between psychological stress, depressive symptoms and MetS, and whether these variables predict MetS and its component trajectories. Material and Methods: Four hundred and twenty-three participants aged $44 \pm 9$ years (59\% women), free of cardiovascular disease at baseline were enrolled into the Chilean study of psychological stress, obesity and MetS. Participants were followed-up for three years (three waves). Each year, they completed psychological questionnaires, anthropometric variables were measured, and blood samples were obtained. Results: Hierarchical linear regression showed that chronic psychological stress at baseline predicted the total number of MetS components (MetS score) during the third assessment wave $(\beta=0.147 ; p<0.01)$. Growth curve modeling allowed to determine that participants who scored +1 standard deviation (SD) at baseline over the mean in psychological stress ( $\beta_{\text {chronicstress }}=0.903 ; 95 \%$ confidence intervals $(C I)=0.065 ; 1.741)$, and depressive symptoms $\left(\beta_{\text {depressive symptoms }}=2.482\right.$; $95 \% C I=0.040 ; 4.923)$ had a higher waist circumference trajectory, as compared to those scoring -1 SD above the mean. Conclusions: Chronic psychological stress is longitudinally associated with the MetS score. Further, psychological stress and depressive symptoms at baseline predicted elevated MetS score trajectories, and a highest waist circumference.

(Rev Med Chile 2018; 146: 1278-1285)

Key words: Depression; Metabolic Syndrome; Stress, Psychological. $\checkmark 1$ 1 síndrome metabólico (SM) es un conjunto de factores de riesgo cardiometabólico que promueven directamente el desarrollo de la enfermedad cardiovascular arteriosclerótica y la diabetes mellitus tipo $2^{1,2}$. Los componentes del SM son la dislipidemia aterogénica, la presión arterial y la glucosa plasmática elevada, proponiéndose, además, a la obesidad abdominal e insulino resistencia como agentes promotores del síndrome. El SM es altamente prevalente en la población chilena. Datos reportados por el Ministerio de Salud de Chile ${ }^{3}$, indican que 35,3\% de los chilenos tienen SM, cifra que es muy similar a la de población norteamericana ${ }^{4}$.
Diversos factores psicológicos están asociados con el SM ${ }^{5}$. De estos, el estrés psicológico, definido como una reacción o respuesta que ocurre cuando un evento o situación excede las capacidades o recursos de adaptación de las personas ${ }^{6-8}$, es una variable que se asocia con $\mathrm{SM}^{9,10}$, tal que personas que experimentan mayor estrés crónico o laboral tienen un mayor riesgo de desarrollar SM y diabetes mellitus tipo $2^{11-13}$. Una asociación similar se observa entre depresión y/o sintomatología depresiva con el SM. Se estima que el riesgo de desarrollar SM es mayor en mujeres que han sufrido un episodio depresivo mayor ${ }^{14,15}$; y en población general se observa un mayor riesgo de presentar 
SM en personas que presentaron depresión mayor en comparación con quienes no lo hicieron, controlando por factores de riesgo cardiovascular clásicos ${ }^{16}$.

Si bien la evidencia internacional demuestra un vínculo entre factores psicológicos y SM, la mayoría de los estudios han sido realizados con muestras norteamericanas y europeas, con participantes caucásicos y/o Latinos que viven en Estados Unidos. En consecuencia, la generalización de estos resultados es una preocupación, dado que el significado e impacto del estrés psicológico varía ampliamente entre culturas ${ }^{17,18}$. Más aún, población latinoamericana ha sido menos investigada en estudios que integren variables psicológicas y desenlaces en salud física. De hecho, son escasas las investigaciones que en la Región hayan examinado la relación entre factores psicológicos y SM. En línea con esto, este estudio que tuvo por objetivo determinar la asociación longitudinal entre estrés psicológico crónico, síntomas depresivos y SM, así como también si estas variables predicen las trayectorias de SM y sus componentes en una muestra de adultos chilenos.

\section{Material y Método}

\section{Participantes}

Este estudio fue realizado con datos obtenidos del estudio chileno de estrés psicológico, obesidad y síndrome metabólico, el cual tuvo un diseño no experimental, longitudinal de panel, que estudió a la misma muestra por tres años $(2015,2016$ y 2017). Se reclutó por medio de un muestreo no probabilístico por conveniencia a 423 trabajadores de la Universidad de La Frontera, Chile. En esta muestra estuvieron representados funcionarios académicos y no académicos (secretarias, auxiliares, guardias, entre otros), quienes cumplieron con los siguientes criterios de inclusión: a) no tener diagnóstico de enfermedad cardiovascular al momento de iniciar el estudio; y b) no haber estado en tratamiento anti-inflamatorio dos semanas previas a la participación en el estudio. Se excluyó a participantes que presentaran enfermedades crónicas tales como algún tipo de cáncer, hipotiroidismo y/o enfermedades inflamatorias.

\section{Instrumentos}

Estrés psicológico: Se utilizó la escala de Brom- berger y Matthews ${ }^{19}$, la cual mide cinco dominios de estrés: Salud personal; salud de un amigo o un familiar; vida laboral; problemas financieros; y relaciones interpersonales con amigos o familiares. Es una escala de respuesta dicotómica $(\mathrm{Si}=$ 1 ; No $=0$ ), que se suma y se obtiene un puntaje total. La confiabilidad de esta escala es alta (Alfa ordinal $=0,90)$.

Sintomatología depresiva: Se utilizó la escala The Center for Epidemiologic Studies Depression Scale (CES-D Scale) ${ }^{20}$. Esta escala consta de 20 ítems, que miden la frecuencia de síntomas depresivos durante la última semana. Las opciones de respuesta son: Raramente o ninguna vez (menos de 1 día $=1)$; pocas veces $(1-2$ días $=2)$, algunas veces (3-4 días $=3$ ) y la mayoría de las veces (5-7 días $=4)$. La confiabilidad obtenida en este estudio fue alta $(\alpha=0,92)$.

Síndrome metabólico: En este estudio se calculó un puntaje total de componentes de SM, sumando la cantidad total de criterios de SM que cada participante cumplió (mínimo 0, máximo 5). Se emplearon los criterios propuestos por el ATP III ${ }^{21}$. El perímetro de cintura, considerado un proxy de obesidad abdominal, fue medido utilizando el procedimiento estándar con una cinta métrica SECA 201 (Seca GmgH \& Co. KG, Hamburg). Las concentraciones de glucosa, colesterol y triglicéridos fueron determinadas por ensayos enzimático-colorimétricos usando kits comerciales convencionales. Se realizaron tres mediciones de presión arterial sistólica y diastólica con un equipo DINAMAP v100 (General Electric). El promedio de la segunda y tercera lectura fue utilizado en este estudio.

Covariables: Los participantes auto-reportaron edad, sexo ( $0=$ hombre; $1=$ mujer $)$, consumo de alcohol $(s i ́=1 ; n o=0)$ y cigarrillos $(s i ́=1 ;$ no $=0)$. El nivel socioeconómico fue estimado a partir del auto-reporte del máximo nivel educacional alcanzado y el nivel de ingresos ${ }^{22}($ bajo $=0$; alto $=1$ ). En adición a estas covariables, en los modelos se controló por variables psicológicas clásicas: Ansiedad e ira rasgo, evaluado con el inventario de personalidad de Spielberg ${ }^{23}$, estrés percibido, medido con la escala de Cohen ${ }^{24}$, percepción de discriminación diaria, dimensionado con la escala de Williams, Yu, Jackson \& Abderson $^{25}$, 
apoyo social, evaluado con la escala $\mathrm{ENRICH}^{26}$ y el optimismo disposicional cuantificado con la escala LOT-R ${ }^{27}$.

\section{Procedimiento}

Durante los tres años de duración del estudio, se siguió el mismo procedimiento, cautelando que el tiempo entre mediciones fuera el mismo para todos los sujetos. Este protocolo de investigación fue aprobado por el Comité Ético Científico de la Universidad de La Frontera. Los participantes fueron contactados vía correo electrónico y telefónicamente. Todos los sujetos asistieron al Laboratorio de Estrés y Salud, con una ayuna mínima de $8 \mathrm{~h}$. Estudiantes de postgrado entrenados, explicaron el propósito del estudio y obtuvieron el consentimiento informado, tras lo cual, se procedió con las medidas antropométricas y de presión arterial. Enfermeras realizaron muestras sanguíneas utilizando un dispositivo BD Vacutainer ${ }^{\circledR}$ UltraTouch $^{\mathrm{TM}}$. Luego de estos procedimientos, los participantes respondieron las encuestas, se les entregó una colación y fueron retribuidos económicamente por su participación. Los resultados de las medidas antropométricas, presión arterial y perfil bioquímico fueron entregados a cada participante.

\section{Análisis estadístico}

Análisis descriptivos y de frecuencias se realizaron para todas las medidas obtenidas en cada uno de los exámenes del estudio (tiempos: línea base, año 2 y año 3 ).

Con la finalidad de estimar la asociación longitudinal entre estrés psicológico crónico, síntomas depresivos (línea base) y el SM y sus componentes (tiempo 3), se realizó análisis de regresión jerárquica, el cual es un tipo de análisis de regresión en el cual un conjunto de variables independientes son ingresadas en pasos secuenciales. El orden de ingreso estas variables está determinado principalmente por el objetivo del estudio ${ }^{28}$, de modo tal que para este análisis, en un primer paso estimó un modelo crudo incorporando las variables de estrés crónico y síntomas depresivos, para posteriormente ir agregando covariables, en los siguientes bloques: 1) "estrés agudo, percepción de discriminación, apoyo social y optimismo disposicional"; 2) "edad, nivel socioeconómico y sexo"; y 3 ) "consumo de alcohol y tabaco".

Con el propósito de estimar si el estrés psi- cológico crónico y la sintomatología depresiva predicen las trayectorias de SM y cada uno de sus componentes, se realizó análisis de curvas de crecimiento, técnica que estima parámetros tradicionales del análisis de regresión (efectos fijos de la constante y pendiente), además de una constante y pendiente aleatorias. La constante aleatoria estima el grado de dispersión en torno al promedio, al comienzo del estudio y la pendiente aleatoria, la variabilidad de los sujetos en torno a la pendiente o trayectoria promedio. En otras palabras permite estimar, la variabilidad tanto en la constante como en la pendiente de cada uno de los sujetos.

El análisis de curvas de crecimiento, permite un manejo flexible de datos perdidos, los cuales, fueron considerados "perdidos al azar" (missed at random $[\mathrm{MAR}])^{29}$. De esta forma los análisis fueron realizados con estimación de máximo verosimilitud con valores perdidos ( $\mathrm{mvml})$, siguiendo las recomendaciones de diversos autores ${ }^{30-32}$.

Todos los análisis fueron realizados con el software STATA 14.2, utilizando un alfa nominal de 0,05 .

\section{Resultados}

Tal como se observa en la Tabla 1, la edad promedio de los participantes en el primer año de estudio (línea base) fue de 44 años (DS $=8,79$ ), con una distribución por sexo de 59\% mujeres y $41 \%$ hombres. El perímetro de cintura promedio para hombres fue $91,93 \mathrm{~cm}(\mathrm{DS}=9,1)$ y para las mujeres 81,29 (DS = 9,9). Solo $24,47 \%$ de la muestra tiene un índice de masa corporal normal, mientras que $49,64 \%$ presenta sobrepeso y $25,89 \%$ tiene obesidad. Se observa heterogeneidad en relación al nivel educacional, con $45 \%$ de la muestra reportando tener enseñanza media o educación técnico-profesional completa y $55 \%$ grado académico de magíster o superior. El $60 \%$ de la muestra reporta un ingreso económico mensual que varía entre 250 mil y 1 millón de pesos.

En relación al análisis de regresión jerárquica, en el modelo crudo se observa que el estrés crónico medido en la línea base, es una variable que predijo de forma estadísticamente significativa el puntaje total de componentes de SM, tal que a mayor estrés crónico el primer año, mayor número de componentes en el tercer año, resultado inalterable en cada uno de los pasos del análisis, controlando 
Tabla 1. Descripción de participantes y componentes del síndrome metabólico

\begin{tabular}{|c|c|c|c|c|c|c|}
\hline & \multicolumn{2}{|c|}{$\begin{array}{c}\text { Año } 1 \text { (2015) } \\
n=423\end{array}$} & \multicolumn{2}{|c|}{$\begin{array}{c}\text { Año } 2(2016) \\
n=373\end{array}$} & \multicolumn{2}{|c|}{$\begin{array}{c}\text { Año } 3(2017) \\
n=379\end{array}$} \\
\hline & $\mathbf{M}$ & DS & M & DS & M & DS \\
\hline Edad & 44,1 & 8,8 & 45,0 & 8,7 & 46,1 & 8,8 \\
\hline Mujer & \multicolumn{2}{|c|}{$58,6 \%$} & \multicolumn{2}{|c|}{$61,5 \%$} & \multicolumn{2}{|c|}{$59,5 \%$} \\
\hline Hombre & \multicolumn{2}{|c|}{$41,4 \%$} & \multicolumn{2}{|c|}{$38,4 \%$} & \multicolumn{2}{|c|}{$40,4 \%$} \\
\hline Glucosa & 93,8 & 27,8 & 87,2 & 26,4 & 92,8 & 21,0 \\
\hline Triglicéridos & 144,9 & 111,8 & 131,1 & 112,2 & 142,8 & 93,4 \\
\hline HDL mujer & 53,8 & 13,1 & 63,3 & 13,3 & 60,1 & 13,5 \\
\hline HDL hombre & 42,8 & 11,3 & 51,2 & 11,7 & 49,0 & 10,9 \\
\hline PA sistólica & 120,3 & 17,6 & 118,9 & 15,8 & 119,7 & 13,2 \\
\hline PA diastólica & 69,4 & 11,5 & 67,8 & 10,7 & 69,4 & 10,6 \\
\hline P. Cintura mujer & 81,3 & 10,2 & 82,4 & 9,9 & 88,8 & 10,0 \\
\hline P. Cintura hombre & 92,5 & 9,9 & 93,5 & 9,4 & 99,0 & 10,7 \\
\hline \% Síndrome metabólico (ATP III) & \multicolumn{2}{|c|}{$20 \%$} & \multicolumn{2}{|c|}{$18,5 \%$} & \multicolumn{2}{|c|}{$17,2 \%$} \\
\hline
\end{tabular}

Fuente: Elaboración propia. $\mathrm{M}$ = promedio; DS = Desviación estándar.

por variables psicológicas, sociodemográficas y del estilo de vida. La sintomatología depresiva no se asoció con el puntaje total de componentes del SM. Se observa que existe una diferencia estadísti- camente significativa entre hombres y mujeres en el número de componentes de SM, y participantes fumadores presentan mayor cantidad de componentes que no fumadores (Tabla 2).

Tabla 2. Predictores de síndrome metabólico

\begin{tabular}{|c|c|c|c|c|c|c|c|c|}
\hline & \multicolumn{2}{|c|}{ Paso 1} & \multicolumn{2}{|c|}{ Paso 2} & \multicolumn{2}{|c|}{ Paso 3} & \multicolumn{2}{|c|}{ Paso 4} \\
\hline & Beta & $\mathbf{p}$ & Beta & $\mathbf{p}$ & Beta & $\mathbf{p}$ & Beta & $\mathbf{p}$ \\
\hline Estrés crónico & 0,160 & 0,006 & 0,161 & 0,004 & 0,152 & 0,007 & 0,147 & 0,009 \\
\hline Síntomas depresivos & $-0,082$ & 0,140 & $-0,083$ & 0,242 & $-0,009$ & 0,892 & $-0,024$ & 0,735 \\
\hline Estrés agudo & & & $-0,124$ & 0,066 & $-0,116$ & 0,082 & $-0,103$ & 0,121 \\
\hline Percepción discriminación & & & 0,049 & 0,397 & 0,022 & 0,699 & 0,031 & 0,594 \\
\hline Apoyo social & & & $-0,020$ & 0,732 & 0,004 & 0,936 & 0,001 & 0,998 \\
\hline Optimismo & & & 0,121 & 0,041 & 0,085 & 0,151 & 0,094 & 0,112 \\
\hline Sexo & & & & & $-0,181$ & 0,001 & $-0,180$ & 0,001 \\
\hline Edad & & & & & 0,081 & 0,130 & 0,076 & 0,155 \\
\hline Nivel socioeconómico & & & & & $-0,008$ & 0,878 & $-0,003$ & 0,944 \\
\hline Bebedor & & & & & & & $-0,062$ & 0,242 \\
\hline Fumador & & & & & & & 0,105 & 0,050 \\
\hline
\end{tabular}

Fuente: Elaboración propia. Paso 1: Modelo crudo sin covariables. Paso 2: Controlando por estrés agudo, percepción de discriminación, apoyo social y optimismo. Paso 3: Variables Paso 1 y paso 2, agregando sexo, edad y nivel socioeconómico. Paso 4: Variables pasos 1, 2, y 3, sumando el estatus de fumador y bebedor. Para la variable sexo, el grupo de referencia son las mujeres. Para el nivel socioeconómico (NSE) el grupo de referencia es el grupo alto. 
Del análisis de regresión jerárquica hecho con cada uno de los componentes del SM (por ejemplo, colesterol HDL, glucosa, triglicéridos), tan sólo en el caso del perímetro de cintura se detecta una asociación estadísticamente significativa con estrés crónico, tal que, a mayor estrés crónico en el primer año, mayor es el perímetro de cintura promedio en el tercer año, controlando por las covariables del estudio.

La Tabla 3, describe los resultados del análisis de curvas crecimiento. En ella se observa que, al comienzo del estudio, los participantes tienen un número promedio de 1,56 componentes, con una variabilidad en torno a este promedio que es estadísticamente significativa, lo que implica que algunos participantes tienen un número mayor de componentes y otros un número menor. En relación a la trayectoria del número de componentes del SM, no existe un cambio estadísticamente significativo en ella, lo cual implica que entre las medidas del estudio el número de componentes promedio de SM se mantiene estable.

En la trayectoria de hombres y mujeres, se observa una diferencia estadísticamente significativa, tal que ellas tienen un menor número de componentes que ellos durante el estudio. Tal como se esperaba, el estrés crónico es una variable que predijo una trayectoria positiva del número promedio de componentes de SM, tal que participantes con mayor puntaje de estrés crónico en la línea base, tienen mayor número de componentes de SM a lo largo del estudio.

En relación a los componentes individuales del SM, se observan resultados estadísticamente significativos sólo en el caso del perímetro de cintura, cuyos promedios en la línea base, examen del segundo y tercer año, fueron respectivamente 92,$5 ; 93,5$ y $99,0 \mathrm{~cm}$ para los varones y 81,29 ; 82,40 y $88,86 \mathrm{~cm}$ para mujeres, existiendo una gran variabilidad en torno a estos promedios. Por cada año que pasa, el perímetro de cintura aumenta en promedio $3,56 \mathrm{~cm}$, cambio en el tiempo que tiene una variabilidad mínima. Se detecta una diferencia en la trayectoria según el sexo, tal que los hombres tienen un mayor perímetro que las mujeres durante todo el estudio. Este resultado es similar para fumadores comparados con no fumadores. Finalmente, se observa que tanto el estrés crónico, como la sintomatología depresiva, se asocian con trayectorias positivas de perímetro de cintura. De esta forma, quienes están una desviación estándar por sobre el promedio (+1 DS) en estrés crónico y/o en síntomas depresivos tienen una trayectoria de perímetro de cintura más elevada durante todo el estudio (Figura 1).

Tabla 3. Trayectorias del número total de componentes de SM y perímetro de cintura

\begin{tabular}{|c|c|c|c|c|c|c|c|c|}
\hline & \multicolumn{4}{|c|}{ Número total de componentes de SM } & \multicolumn{4}{|c|}{ Perímetro de cintura } \\
\hline & $\beta$ & $\mathbf{p}$ & $95^{\circ}$ & C.I. & $\beta$ & $\mathbf{p}$ & 95 & o C.I. \\
\hline Estrés crónico & 0,146 & 0,006 & 0,043 & 0,250 & 0,903 & 0,035 & 0,065 & 1,741 \\
\hline Síntomas depresivos & 0,052 & 0,684 & $-0,202$ & 0,307 & 2,482 & 0,046 & 0,040 & 4,923 \\
\hline Constante & 1,564 & 0,000 & 0,838 & 2,291 & 94,638 & 0,000 & 86,403 & 102,873 \\
\hline \multicolumn{9}{|l|}{ Efectos aleatorios } \\
\hline$\sigma^{2}$ trayectoria & 0,008 & - & 0,000 & 7,613 & 0,001 & - & 0,000 & 3577,049 \\
\hline$\sigma^{2}$ constante & 0,842 & - & 0,665 & 1,067 & 74,25 & - & 62,882 & 87,683 \\
\hline$\sigma^{2}$ trayectoria $X$ constante & $-0,077$ & - & $-0,162$ & 0,006 & 0,286 & - & $-2,369$ & 2,941 \\
\hline$\sigma^{2}$ Residuo & 0,567 & - & 0,488 & 0,658 & 16,186 & - & 14,560 & 17,994 \\
\hline
\end{tabular}

Fuente: Elaboración propia. En el apartado de efectos fijos, la constante representa el promedio de número de componentes del síndrome en la línea base. En el apartado de efectos aleatorios, la $\sigma^{2}$ constante representa la variabilidad en torno al promedio del número de componentes de síndrome en la línea base. 


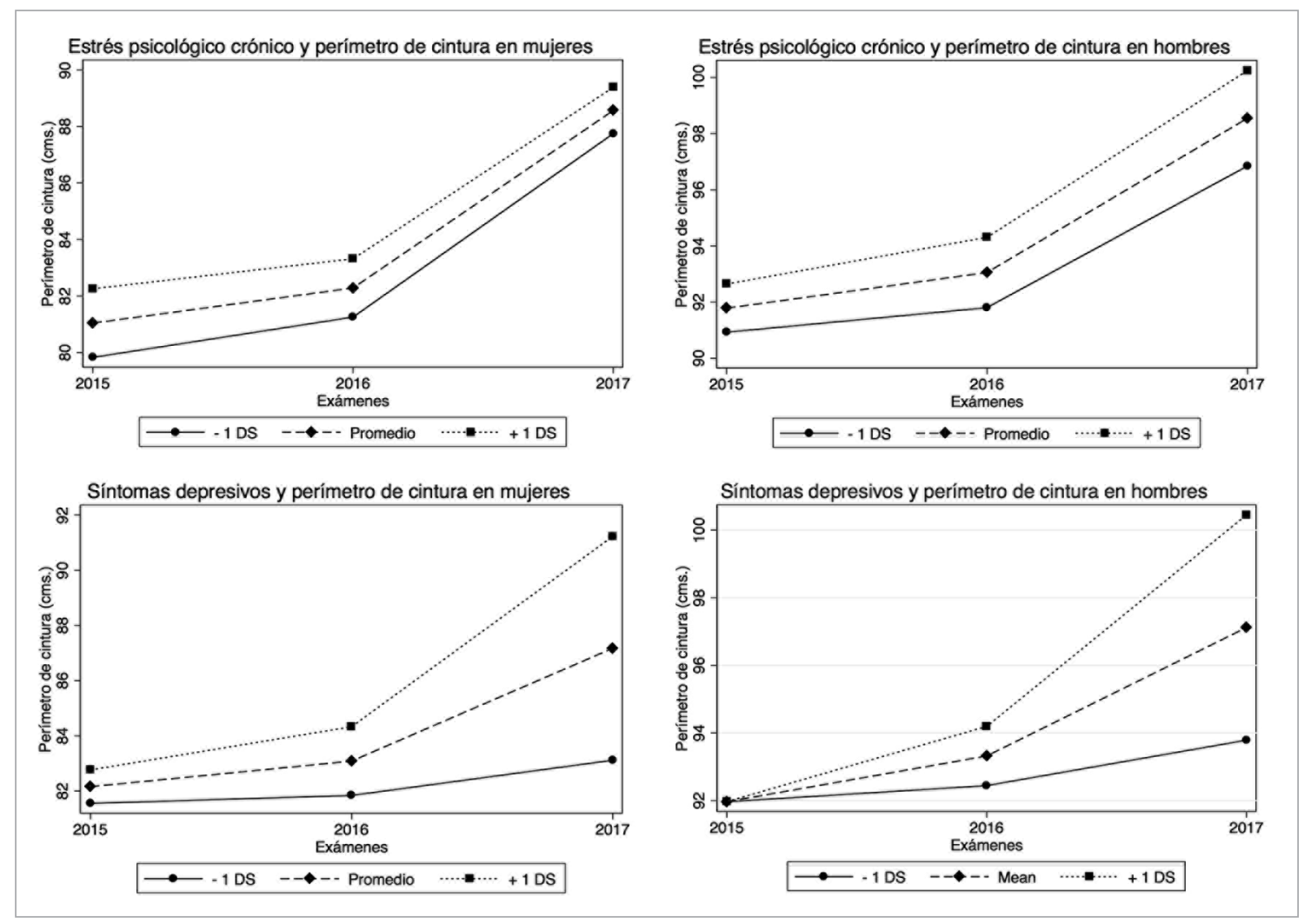

Figura 1. Trayectorias de perímetro de cintura según estrés psicológico crónico y síntomas depresivos, en mujeres y hombres. Fuente: Elaboración propia. En los gráficos se observa que la trayectoria promedio de perímetro de cintura aumenta desde la línea base (2015), hasta el tercer examen (2017). Quienes puntúan 1 desviación estándar por sobre el promedio en estrés crónico y en síntomas depresivos (+1 DS), tienen una trayectoria más elevada de perímetro de cintura que quienes puntúan 1 desviación por bajo el promedio (-1 DS).

\section{Discusión}

En este estudio, el estrés psicológico crónico se asoció en forma longitudinal con un puntaje total de número de componentes $\mathrm{SM}$, resultado robusto controlando por variables psicológicas clásicas (ansiedad e ira, discriminación diaria, apoyo social y optimismo disposional), variables sociodemográficas (edad, sexo, nivel socioeconómico) y de estilo de vida (tabaquismo, consumo de alcohol). En participantes que autorreportan mayor estrés crónico, se observa una trayectoria en el tiempo que tiende a un mayor número de componentes de SM. Al realizar el análisis con cada uno de los componentes individuales del síndrome, sólo en el caso del perímetro de cintura, se observa una trayectoria más elevada entre quienes tienen mayor estrés crónico.
Estos resultados son consistentes con estudios internacionales ${ }^{11,13}$, y destacan el correlato fisiológicos del estrés psicológico, el cual se caracteriza por la desregulación del sistema cardiovascular y el eje hipotalámico hipofisiario adrenal $(\mathrm{HHA})^{33}$, hiperglicemia ${ }^{34}$, aumento de la presión arterial, circulación de lípidos, glucosa e inflamación y finalmente aumento del depósito de grasa abdominal ${ }^{35}$.

Los resultados de este estudio relevan el rol de las variables psicológicas en el riesgo de desarrollar SM. Dado que al comienzo del estudio los participantes estuvieron libres de enfermedad cardiovascular, es posible evidenciar un posible vínculo causal entre estrés psicológico y el SM. En particular, se observa una asociación longitudinal entre estrés crónico y síntomas depresivos con el perímetro de cintura, lo cual permite hipotetizar 
que la relación entre estas variables psicológicas y los otros componentes del síndrome podría estar mediada por el perímetro de cintura.

Los resultados de este estudio, tiene implicancias en la comprensión de cómo variables psicológicas y fisiológicas se relacionan e impactan en salud física. Además, generan oportunidades para diseñar intervenciones que eduquen a afrontar exitosamente el estrés crónico y los síntomas depresivos.

Este estudio tiene fortalezas y limitaciones. Entre las primeras, están el diseño longitudinal e integración de variables psicológicas y fisiológicas. $\mathrm{El}$ análisis estadístico empleado permite modelar datos longitudinales y la imputación de casos perdidos. Dentro de las limitaciones, están la no incorporación de medidas de dieta, actividad física, y respuestas fisiológicas al estrés (cortisol o marcadores de inflamación), pues existe evidencia que todas estas variables pueden mediar el vínculo entre estrés psicológico, obesidad y síndrome metabólico ${ }^{36-38}$. El muestreo no probabilístico empleado en este estudio, supone tener cautela a la hora de generalizar resultados a la población general.

\section{Conclusión}

En este estudio el estrés psicológico se asoció longitudinalmente con el número de componentes del SM, una muestra de adultos libres de enfermedad cardiovascular declarada. Este resultado releva el rol de variables psicológicas en la génesis del síndrome, y genera oportunidades de intervención de factores de riesgo cardiovascular no clásicos.

Agradecimientos: Este estudio fue financiado por la Comisión Nacional Científica y Tecnológica de Chile, por medio de un proyecto FONDECYT de Iniciación 1140454, cuyo investigador principal fue el Dr. Manuel S. Ortiz.

\section{Referencias}

1. Alberti KGMM, Eckel RH, Grundy SM, Zimmet PZ, Cleeman JI, Donato KA, et al. Harmonizing the metabolic syndrome. Circulation 2009; 120: 1640-5.

2. Eckel RH, Alberti KGMM, Grundy SM, Zimmet PZ. The metabolic syndrome. Lancet 2010; 375 (9710): 181-3.
3. INE. Encuesta Nacional de Salud [Internet]. Instituto Nacional de Estadística. 2010. Available from: http:// www.ine.es/dyngs/INEbase/es/operacion.htm?c=Estadistica_C\&cid $=1254736176783 \&$ menu $=$ resultados\&sec$\mathrm{c}=1254736194724$ \&idp=1254735573175.

4. Beltrán-Sánchez H, Harhay MO, Harhay MM, McElligott S. Prevalence and Trends of Metabolic Syndrome in the Adult U.S. Population, 1999-2010. J Am Coll Cardiol. Elsevier Ltd 2013; 62 (8): 697-703.

5. Ortiz MS, Willey JF, Chiang JJ. How stress gets under the skin. Rev Med Chile 2014; 142 (6): 451-7

6. Cohen S. Social relationships and health. Am Psychol 2004; 59: 676-84.

7. Cohen S. Perceived stress scale. Psychology 1994;1-3.

8. Cohen S, Janicki-Deverts D, Miller GE. Psychological stress and disease. Jama 2007; 298 (14): 1685-7.

9. Vitaliano PP, Scanlan JM, Zhang J, Savage M V, Hirsch IB, Siegler IC. A path model of chronic stress, the metabolic syndrome, and coronary heart disease. Psychosom Med 2002; 64 (3): 418-35.

10. Bove M, Carnevali L, Cicero AFG, Grandi E, Gaddoni M, Noera G, et al. Psychosocial factors and metabolic parameters: is there any association in elderly people? The Massa Lombarda Project. Aging Ment Health 2010; 14 (7): 801-6.

11. Chandola T, Brunner E, Marmot M. Chronic stress at work and the metabolic syndrome: prospective study. BMJ 2006; 332 (7540): 521-5.

12. Heraclides AM, Chandola T, Witte DR, Brunner EJ. Work Stress, Obesity and the Risk of Type 2 Diabetes: Gender-Specific Bidirectional Effect in the Whitehall II Study. Obesity 2012; 20 (2): 428-33.

13. Ortiz MS, Myers HF, Schetter CD, Rodríguez CJ, Seeman TE. Psychosocial predictors of metabolic syndrome among Latino groups in the Multi-Ethnic Study of Atherosclerosis (MESA). PLoS One. Public Library of Science 2015; 10 (4).

14. Goldbacher EM, Bromberger J, Matthews KA. Lifetime history of major depression predicts the development of the metabolic syndrome in middle-aged women. Psychosom Med 2009; 71 (3): 266-72.

15. Goldbacher EM, Matthews KA, Goldbacher Karen A. EMM. Are psychological characteristics related to risk of the metabolic syndrome? A review of the literature. Ann Behav Med 2007; 34 (3): 240-52.

16. Butnoriene J, Bunevicius A, Norkus A, Bunevicius R. Depression but not anxiety is associated with metabolic syndrome in primary care based community sample. Psychoneuroendocrinology. Elsevier 2014; 40 (1): 26976.

17. Laungani P, Adler LL, Gielen UP. The influence of 
culture on stress: India and England. In: Cross-cultural topics in psychology (2nd ed). 2001. p. 149-69.

18. Markus HR, Kitayama S. Culture and the self: Implications for cognition, emotion, and motivation. Psychol Rev 1991; 98 (2): 224-53.

19. Bromberger JT, Matthews KA. A longitudinal study of the effects of pessimism, trait anxiety, and life stress on depressive symptoms in middle-aged women. Psychology and Aging 1996; 11: 207-13.

20. Radloff LS. A Self-Report Depression Scale for Research in the General Population. Appl Psychol Meas 1977; 1 (3): 385-401.

21. Alberti KGMM, Zimmet P, Shaw J. Metabolic syndrome--a new world-wide definition. A Consensus Statement from the International Diabetes Federation. Diabet Med 2006; 23 (5): 469-80.

22. Adimark. Mapa socioeconómico de Chile. 2002. Available from: http:/www.adimark.cl/medios/estudios/ informe_mapa_socioeconomico_de_chile.pdf

23. Spielberger CD, Sydeman SJ. State-trait anxiety inventory and state-trait anger expression inventory. In: The use of psychological testing for treatment planning and outcome assessment 1994. p. 292-321.

24. Cohen S, Kamarck T, Mermelstein R. A Global Measure of Perceived Stress. J Health Soc Behav 1983; 24 (4): 385-96.

25. Williams DR, Yu Y, Jackson JS, Anderson NB. Racial differences in physical and mental health: Socio-economic status, stress and discrimination. J Health Psychol 1997; 2 (3): 335-51.

26. ENRICHD Investigators. Enhancing Recovery in Coronary Heart Disease (ENRICHD) study intervention: rationale and design. Psychosom Med 2001; 63 (5): 747-55.

27. Ortiz MS, Gómez-Pérez D, Cancino M, Barrera-Herrera A. Validación de la versión en Español de la Escala de Optimismo Disposicional (LOT-R) en una muestra Chilena de estudiantes universitarios. Ter Psicol 2016;
34 (1): 53-8.

28. Cohen J, Cohen P, West SG, Aiken LS. Applied multiple regression/correlation analysis for the behavioral sciences. New Jersey: Lawrence Erlbaum Associates; 2003.

29. Little TD. Longitudinal structural equation modeling. New York: The Guildoford Press. 2013.

30. Hox J. Multilevel analysis - Techniques and applications. Multilevel analysis techniques and applications. New York: Routledge; 2010.

31. Cnaan A, Laird NM, Slasor P. Using the general linear mixed model to analyse unbalanced repeated measures and longitudinal data. Stat Med 1997; 16 (20): 234980.

32. Raudenbush SW, Bryk, A. Hierarchical linear models. Applications and data analysis methods. London: Sage Publications; 2002.

33. Kibler JL, Tursich M, Ma M, Malcolm L, Greenbarg R. Metabolic, autonomic and immune markers for cardiovascular disease in posttraumatic stress disorder. World J Cardiol 2014; 6 (6): 455.

34. Brunner EJ, Hemingway H, Walker BR, Page M, Clarke $\mathrm{P}$, Juneja $\mathrm{M}$, et al. Adrenocortical, autonomic, and inflammatory causes of the metabolic syndrome: Nested case-control study. Circulation 2002; 106 (21): 2659-65.

35. Epel ES. Psychological and metabolic stress: A recipe for accelerated cellular aging? Hormones 2009; 8: 7-22.

36. Tomiyama A, Finch L, Cummings J. Did That Brownie Do Its Job? Stress, Eating, and the Biobehavioral Effects of Comfort Food. Emerg Trends Soc Behav Sci 2015; $1-15$.

37. Hamer M. Psychosocial Stress and Cardiovascular Disease Risk: The Role of Physical Activity. Psychosom Med 2012; 74 (9): 896-903.

38. Kaptoge S, Di Angelantonio E, Lowe G, Pepys MB, Thompson SG, Collins R, et al. C-reactive protein concentration and risk of coronary heart disease, stroke, and mortality: an individual participant meta-analysis. Lancet 2010; 375 (9709): 132-40. 